\title{
The effect of the contaminant emission rate on the velocity field and contaminant distribution with the presence of an obstacle in a large space
}

\author{
Qianru Zhang ${ }^{1}$, Chengqiang Zhi ${ }^{1}$, Yixiang Huang ${ }^{1}$, Wei Ye ${ }^{1}$, Jun Gao ${ }^{1}$ and Xu Zhang ${ }^{1, *}$ \\ ${ }^{1}$ School of Mechanical Engineering, Tongji University, Shanghai 201804, PR China
}

\begin{abstract}
In the industrial field, the prediction of the contaminant gas distribution is very meaningful. However, when the leakage is high, not only the contaminant distribution will not follow the pattern of the original flowfield, but the contaminant buoyancy or negative buoyancy will affect the flowfield conversely. In this study, we focus on the effect of the contaminant emission rate on the velocity field and contaminant distribution with an obstacle in a large space by means of CFD simulation. Two leaking positions and five emission rates of the source have been taken into consideration. When the emission rate is high enough, the flowfield structure will be altered and new vortexes will appear. The contaminant dimensionless concentration distribution is totally different from the low-emission-rate conditions. The flammable region becomes significant, which leads to the potential risk of explosion.
\end{abstract}

\section{Introduction}

It is unrealistic to assume that the pollutant concentration is well-mixed due to the dimensions for large spaces. The significant spatial variations in contaminant concentration have been investigated in many studies in manufacturing plants and other laboratory experiments[1-5]. However, the effect of gas buoyancy is rarely taken into account, which will influence the flowfield and the concentration field. Obstacles usually exist in realistic plants. The windward and leeward sides of the obstacles are usually featured with small velocities and vortexes due to the surrounding flow patterns[67]. When the leakages occur in such areas, it's easy to cause accumulation. Many studies have been done concerned with the concentration distribution with the existence of obstacles in the atmospheric field[6, 8-9]. However, the flow patterns in enclosed spaces are quite different from the atmospheric incoming flow and are affected by the space configuration, supply and exhaust positions, supply or exhaust velocities, etc. 
Buoyancy strength is related to the contaminant emission rate and the environmental velocity field[10]. When the emission rate is small with regard to the nearby velocity, the contaminant gas can be regarded as passive and transport with the airflow. When the emission rate is high to some extent, the effect of the density starts to appear. In this study, we simulate the contaminant gas leaked at two sides of the obstacle with sulfur hexafluoride $\left(\mathrm{SF}_{6}\right)$ as the contaminant gas in the same ventilation mode by CFD. The effect of the buoyancy on the flow field and concentration field are discussed. The critical range in which the gas is taking a noticeable effect on the flowfield and the concentration field.

\section{Methodology}

\subsection{Domain, computational grid, and boundary conditions}

We investigate a $5 \mathrm{~m}(\mathrm{X}) * 6 \mathrm{~m}(\mathrm{Y}) * 6 \mathrm{~m}(\mathrm{Z})$ space with a $1 \mathrm{~m}^{*} 1 \mathrm{~m} * 1 \mathrm{~m}$ cubic obstacle at the center of the floor. The air is supplied from the middle of the $5 \mathrm{~m}$-wide wall and exhausted from the bottom of the opposite wall. The air change rate of the space is $3 h^{-1}$. The air jet first impinges the opposite wall and then spreads and recirculates. We designate the space between the obstacle and the wall of the outlet as A side and the other side as B side. As for the contaminant leakage location, one is $0.5 \mathrm{~m}$ from the obstacle on A side and $0.5 \mathrm{~m}$ above the floor, denoted by $\mathrm{A}(0,4 \mathrm{~m}, 0.5 \mathrm{~m})$, the other is $0.5 \mathrm{~m}$ from the obstacle on B side and $0.5 \mathrm{~m}$ above the floor, denoted by $\mathrm{B}(0,2 \mathrm{~m}, 0.5 \mathrm{~m})$. We assume the contaminant source is a point source.

We built half the space, a $2.5 \mathrm{~m} * 6 \mathrm{~m} * 6 \mathrm{~m}$ space, in ANSYS Geometry Modeler, as shown in Fig.1. The symmetry plane is highlighted with blue. The air supply is in the center of the wall, the air exhaust is $0.2 \mathrm{~m}$ above the floor on the opposite wall. The diameter of the supply and exhaust openings is $0.12 \mathrm{~m}$. For air supply, velocity inlet with $13 \mathrm{~m} / \mathrm{s}$ is set. For air exhaust, pressure outlet with a gauge pressure of 0 is set. The contaminant source in the model is a cubic zone.

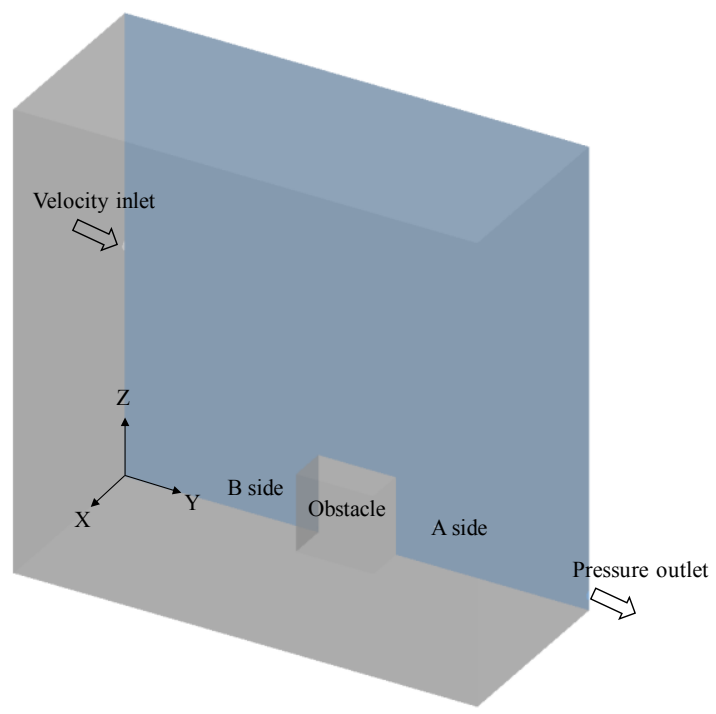

Fig.1. The space built in Geometry Modeler.

\subsection{Computational setup}

The commercial software ANSYS FLUENT is used for the steady RANS computations based on a control volume approach for solving flow and mass fraction equations. The Green-Gauss cellbased scheme is used for gradient discretization. The advection terms are discretized using a second-order upwind scheme. The semi-implicit method for the pressure-linked equation (SIMPLE) algorithm is used for the pressurevelocity coupling.

The spatial distribution of airflow, temperature, and species in the zone is governed by the conservation laws of mass, momentum, and energy. The governing advection-diffusion equations of the fluid are all in the form of[11]:

$\frac{\partial \rho \varphi}{\partial t}+\operatorname{div}(\rho \vec{U} \varphi)=\operatorname{div}\left(\Gamma_{\varphi} \overrightarrow{\operatorname{grad} \varphi}\right)+S_{\varphi}$

Where $\rho$ is the density of the fluid, $\varnothing$ is the scalar under discussion, $t$ is time, $\vec{U}$ is the velocity vector, $\Gamma_{\varnothing}$ is the diffusion coefficient, $S_{\emptyset}$ is the source term.

The dependent variables, effective diffusion coefficients and the source terms for each equation are concluded in Table 1[11]: 
Table 1. The dependent variables, effective diffusion coefficients and the source terms in the transport equation.

\begin{tabular}{|c|c|c|c|}
\hline Equation & $\varphi$ & $\Gamma_{\varphi}$ & $S_{\varphi}$ \\
\hline Continuity & 1 & 0 & 0 \\
\hline Momentum X & $u$ & $\mu$ & $-\frac{\partial P}{\partial x}$ \\
\hline Momentum Y & $v$ & $\mu$ & $-\frac{\partial P}{\partial y}$ \\
\hline Momentum Z & $w$ & $\mu$ & $-\frac{\partial P}{\partial z}$ \\
\hline Energy & $C_{p} T$ & $\lambda$ & $Q$ \\
\hline Concentration & $Y_{i}$ & $d$ & $Q_{m}$ \\
\hline
\end{tabular}

Where $u, v, w$ are respectively the velocities in $\mathrm{X}$, $\mathrm{Y}$, and $\mathrm{Z}$ direction; $T$ is the temperature; $C_{p}$ is the specific heat; $Y_{i}$ is the species mass fraction; $\mu$ is the kinetic viscosity; $\lambda$ is the temperature conduction coefficient; $d$ is the mass transfer coefficient; $P$ is the pressure; $g$ is the gravitational acceleration; $\rho$ is the density; $Q$ is the energy generated by the source; $Q_{m}$ is the contaminant strength generated by the source.

The local mass fraction of the species, $Y_{i}$, is calculated by solving a convection-diffusion equation for the species with the parameter in the last row of Table 1.

Since the study here is focused on the effect of the negative buoyancy on the flow field and the concentration field, we only use the standard $k-\varepsilon$ model as the turbulence model and standard wall function as the near wall treatment. The $\mathrm{y}+$ values are kept in the range of 30-300.

\subsection{Configurations}

We use two contaminant source locations in this study as mentioned before. For each source location, there are five emission rates. All the configurations are listed in Table 2.

Table 2. The simulation configurations.

\begin{tabular}{|c|c|c|c|}
\hline Configuration & $\begin{array}{c}\text { Air } \\
\text { change } \\
\text { rate }\left(\mathrm{h}^{-1}\right)\end{array}$ & $\begin{array}{c}\text { Emission } \\
\text { location } \\
\text { (m) }\end{array}$ & $\begin{array}{l}\text { Emission } \\
\text { strength } \\
\left(\mathrm{kg} / \mathrm{m}^{3} / \mathrm{s}\right)\end{array}$ \\
\hline $\mathrm{A} 1$ & \multirow{5}{*}{3} & \multirow{5}{*}{$\begin{array}{c}(0,4 \\
0.5)\end{array}$} & 1 \\
\hline $\mathrm{A} 2$ & & & 5 \\
\hline A3 & & & 10 \\
\hline A4 & & & 20 \\
\hline A5 & & & 50 \\
\hline B1 & \multirow{5}{*}{3} & \multirow{5}{*}{$\begin{array}{c}(0,2 \\
0.5)\end{array}$} & 1 \\
\hline B2 & & & 5 \\
\hline B3 & & & 10 \\
\hline B4 & & & 20 \\
\hline B5 & & & 50 \\
\hline
\end{tabular}

\subsection{Indexes for the contaminant distribution} and the negative buoyancy strength

As the emission rate changes in different simulation configurations, we need a dimensionless concentration to judge the contaminant distribution. The dimensionless concentration $C^{*}$ is defined as:

$C^{*}=\frac{C}{C_{0}}$

$C_{0}=\frac{q}{Q_{V}}$

Where $C$ is the concentration of $\mathrm{SF}_{6}, \mathrm{~kg} / \mathrm{m}^{3} ; C_{0}$ is contaminant concentration when the room is fully mixed, $\mathrm{kg} / \mathrm{m}^{3} ; q$ is contaminant emission rate, $\mathrm{kg} / \mathrm{s} ; Q_{V}$ is room ventilation rate, $\mathrm{m}^{3} / \mathrm{s}$.

We also need a criterion to judge the negative buoyancy strength, which is:

$\theta=\left(\frac{g \Delta \rho}{\rho} \frac{q}{D}\right)^{1 / 3} / U_{e}$

Where $\theta$ is the dimensionless number to discriminate passive gas originally[10]; $g$ is the gravitational acceleration, $\mathrm{kg} / \mathrm{m}^{2} / \mathrm{s} ; \Delta \rho$ is density difference between the contaminant gas and the environment gas, $\mathrm{kg} / \mathrm{m}^{3} ; \rho$ is environment gas density, $\mathrm{kg} / \mathrm{m}^{3} ; \quad D$ is contaminant source characteristic dimension. 
The dimensionless number $\theta$ was proposed by Britter[10] as a criterion to determine whether the contaminant gas is passive or not. Here we further the definition and inspect the how it affects the flowfield.

Table 3 gives the $\theta$ values with different emission rates when the source is at $(0,4 \mathrm{~m}, 0.5 \mathrm{~m})$ and $(0$, $2 \mathrm{~m}, 0.5 \mathrm{~m}$ ) respectively.

Table 3. $\theta$ values in all the configurations.

\begin{tabular}{|c|c|c|c|c|}
\hline & \multicolumn{2}{|c|}{$\mathrm{A}(0,4 \mathrm{~m}, 0.5 \mathrm{~m})$} & \multicolumn{2}{c|}{$\mathrm{B}(0,2 \mathrm{~m}, 0.5 \mathrm{~m})$} \\
\hline $\begin{array}{c}\text { source } \\
\text { emission } \\
\text { rate } \\
\left(\mathrm{kg} / \mathrm{m}^{3} / \mathrm{s}\right)\end{array}$ & $\theta$ & $C_{0}\left(\mathrm{~kg} / \mathrm{m}^{3}\right)$ & $\theta$ & $C_{0}\left(\mathrm{~kg} / \mathrm{m}^{3}\right)$ \\
\hline 1 & 1.45 & 0.0001 & 2.701 & 0.0001 \\
\hline 5 & 2.479 & 0.0005 & 4.618 & 0.0005 \\
\hline 10 & 3.124 & 0.0011 & 5.819 & 0.0011 \\
\hline 20 & 3.936 & 0.0021 & 7.331 & 0.0021 \\
\hline 50 & 5.342 & 0.0053 & 9.95 & 0.0053 \\
\hline
\end{tabular}

\subsection{The flammable region}

The contaminant gases are probably flammable in many industrial occasions. Since the flammable limits for different flammable gases are different, here we adopt $1 \%$ as the lower explosive limit for the contaminant gas under investigation. Most safety authorities and fire underwriters prefer to limit concentrations to $20 \%$ to $25 \%$ of the lower explosive limit of a material. In this study, the flammable region is defined as the area with the volumetric concentration exceeding $20 \%$ of the lower explosive limit, which is $2000 \mathrm{ppm}$.

\section{Results and discussion}

\subsection{Flowfield}

Fig.2 Fig.4 give the velocity magnitude contours and the streamlines of the $\mathrm{z}=0.5 \mathrm{~m}$ plane, the $\mathrm{z}=0.2 \mathrm{~m}$ plane and the symmetry plane without contaminant gas emission. The contours are mirrored by the symmetry plane and displayed. While the streamlines are drawn only in the computational domain.

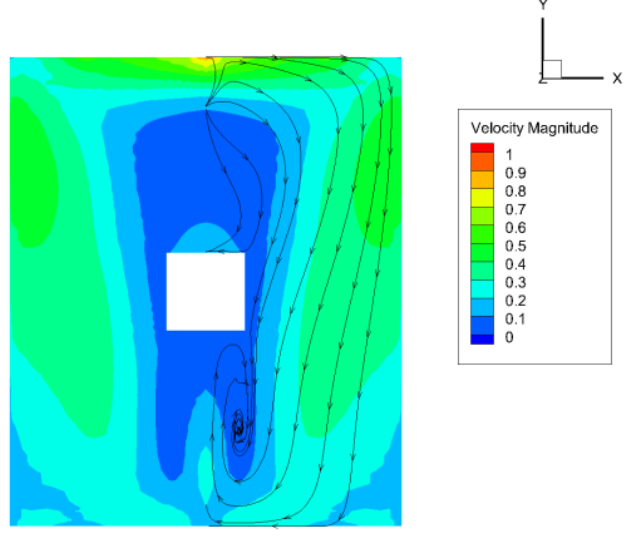

Fig.2. Velocity magnitude contours and streamlines of the $\mathrm{z}=0.5 \mathrm{~m}$ plane without contaminant gas emission.
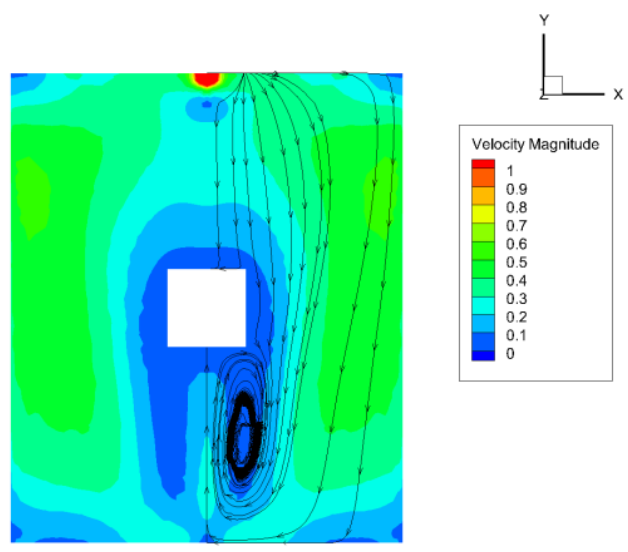

Fig.3. Velocity magnitude contours and streamlines of the $\mathrm{z}=0.2 \mathrm{~m}$ plane without contaminant gas emission.

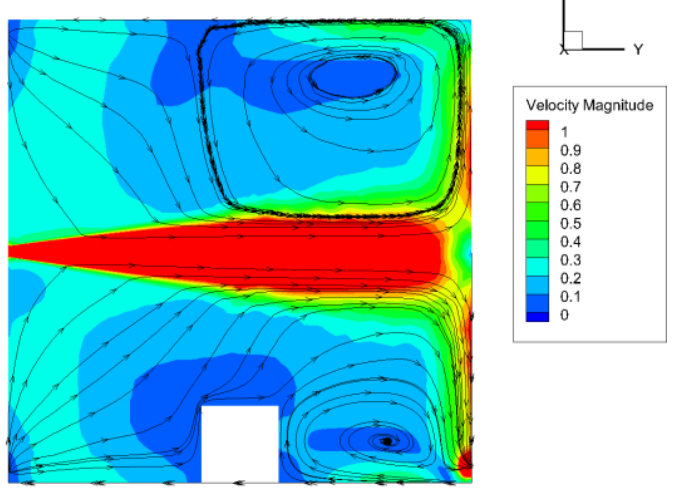


Fig.4. Velocity magnitude contours and streamlines of the symmetry plane without contaminant gas emission.

In Fig. 2 and Fig.3, after the flow passing the obstacle, a vortex is formed, which coincides with the previous studies about flow passing obstacles. In Fig.4, due to the high velocity of the inlet jet, after impinging on the opposite wall, it spreads towards all the direction and causes a vortex between the outlet wall and the obstacle. The velocity at $\mathrm{A}(0,4 \mathrm{~m}, 0.5 \mathrm{~m})$ and $\mathrm{B}(0,2 \mathrm{~m}, 0.5 \mathrm{~m})$ are lower than the surrounding environment.

Fig.5 Fig. 7 give the velocity magnitude contours and the streamlines of the $\mathrm{z}=0.5 \mathrm{~m}$ plane, the

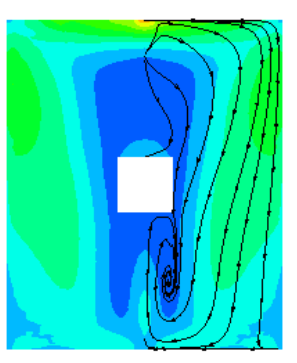

A1

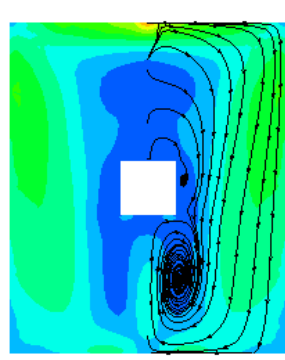

A4
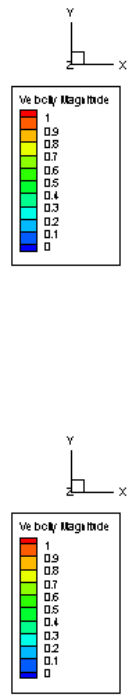

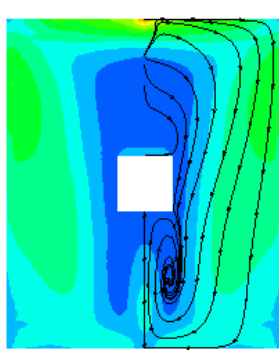

A2

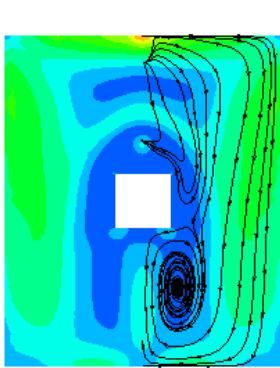

A5 $\mathrm{z}=0.2 \mathrm{~m}$ plane and the symmetry plane with the contaminant gas released at $\mathrm{A}(0,4 \mathrm{~m}, 0.5 \mathrm{~m})$. With the negative buoyancy strength increasing, the low-velocity zone, in which velocity is from 0 to $0.1 \mathrm{~m} / \mathrm{s}$, tends to move towards the B side of the obstacle. The structures of the flowfield for the $\mathrm{z}=0.5 \mathrm{~m}$ plane and $\mathrm{z}=0.2 \mathrm{~m}$ plane both change dramatically from A4 to A5 (from $\theta=3.936$ to $\theta=5.342$ ). As for the streamlines, we can observe that the vortex at the $\mathrm{B}$ side of the obstacle become larger. The small circle zone in A5 in which velocity is from $0.1 \mathrm{~m} / \mathrm{s}$ to $0.3 \mathrm{~m} / \mathrm{s}$ is due to the negative buoyancy becoming noticeable and the vertical velocity increases to some extent.

Fig.5. Velocity magnitude field of the $z=0.5 \mathrm{~m}$ plane with contaminant gas released at $A(0,4 \mathrm{~m}, 0.5 \mathrm{~m})$. 

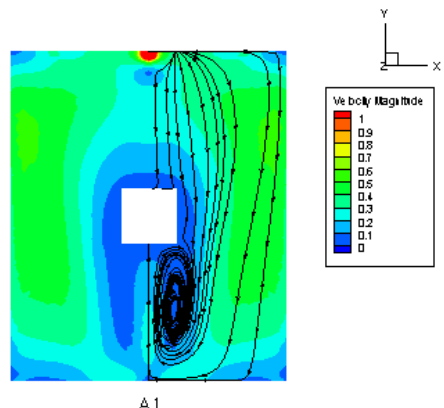

A1

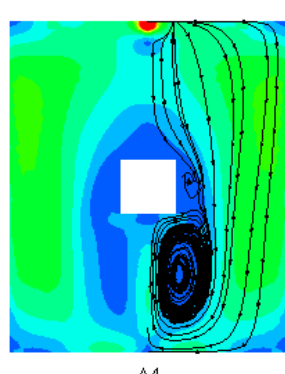

A4

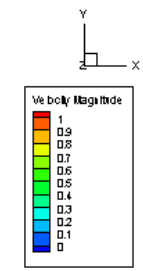

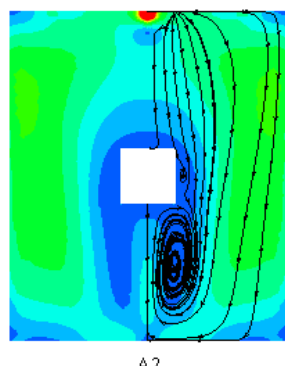
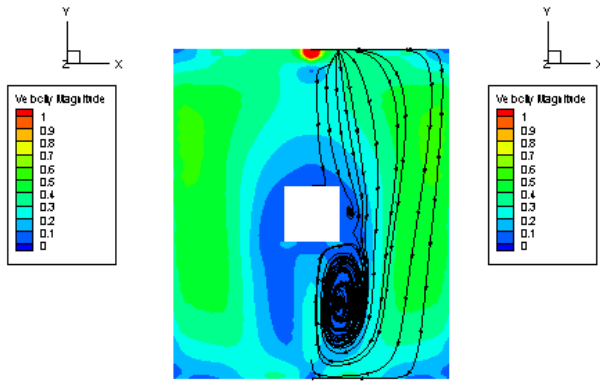
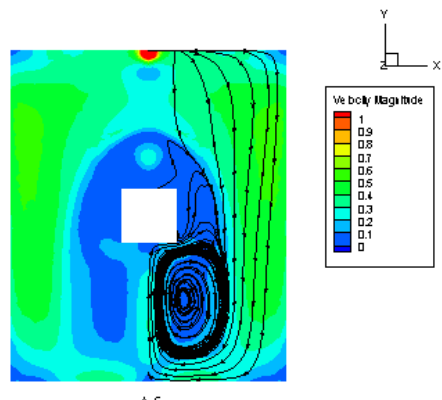

Fig.6. Velocity magnitude field of the $\mathrm{z}=0.2 \mathrm{~m}$ field with contaminant gas released at $\mathrm{A}(0,4 \mathrm{~m}, 0.5 \mathrm{~m})$.

Fig. 7 gives the velocity magnitude contours of the symmetry plane. We can observe that with the increase of $\theta$, the single vortex gradually changes on the A side of the obstacle. In the A4 $(\theta=3.936)$ and A5 $(\theta=5.342)$ condition, a new vortex starts to emerge.

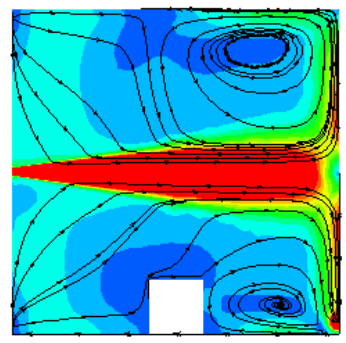

A1

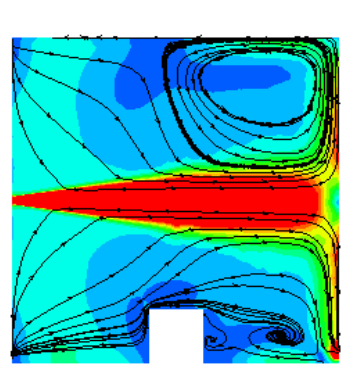

A4
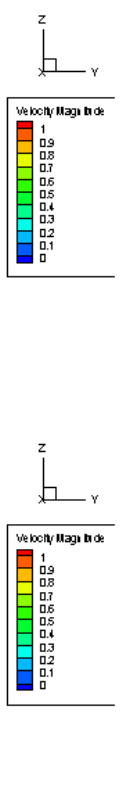

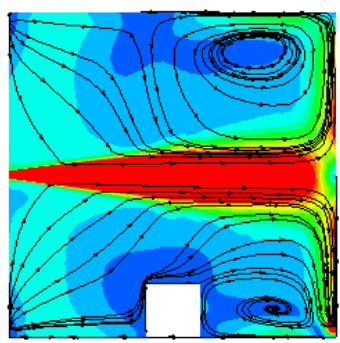

A2

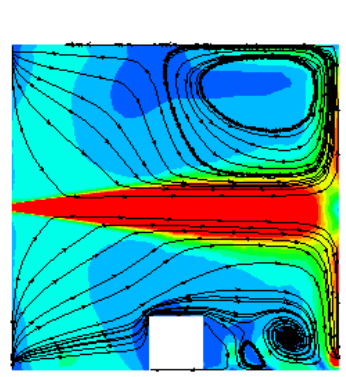

A5
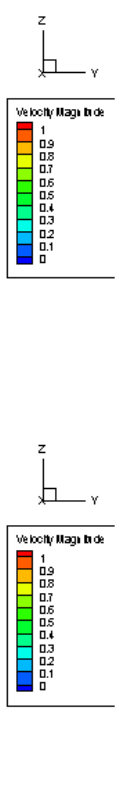

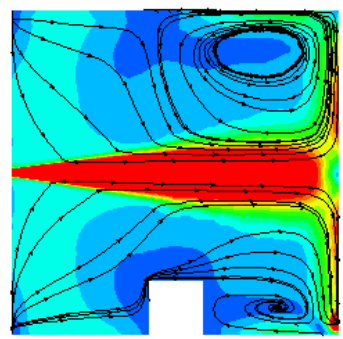

A3

Fig.7. Velocity magnitude field of the symmetry plane with contaminant gas released at $\mathrm{A}(0,4 \mathrm{~m}, 0.5 \mathrm{~m})$.

Fig.8 Fig.10 give the velocity magnitude contours and the streamlines of the $\mathrm{z}=0.5 \mathrm{~m}$ plane, the $\mathrm{z}=0.2 \mathrm{~m}$ plane and the symmetry plane with the contaminant gas released at $\mathrm{B}(0,2 \mathrm{~m}, 0.5 \mathrm{~m})$. Since the contaminant source is at the B side of the obstacle, where the vortexes exist. With the 
negative buoyancy strength increasing, the lowvelocity zone, in which velocity is from 0 to $0.2 \mathrm{~m} / \mathrm{s}$, tends to move outward from the symmetry plane. The structure of the flowfield for
$\mathrm{Z}=0.5 \mathrm{~m}$ plane changes from $\mathrm{B} 4$ to $\mathrm{B} 5$ (from $\theta=7.331$ to $\theta=9.950$ ). The structure of the flowfield for $\mathrm{z}=0.2 \mathrm{~m}$ plane changes from $\mathrm{B} 3$ to B4 (from $\theta=5.819$ to $\theta=7.331$ ).

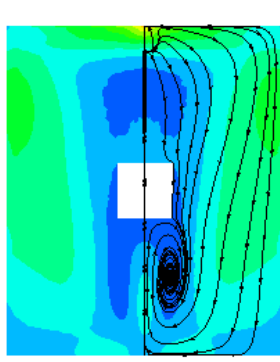

B1

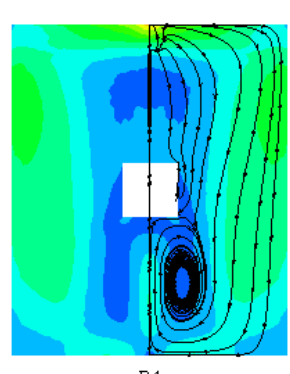

B4
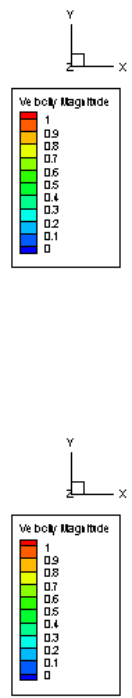

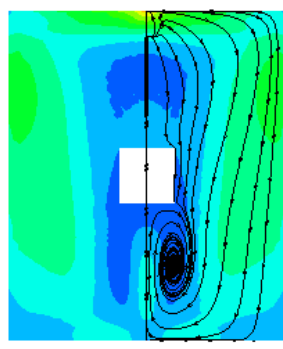

B2

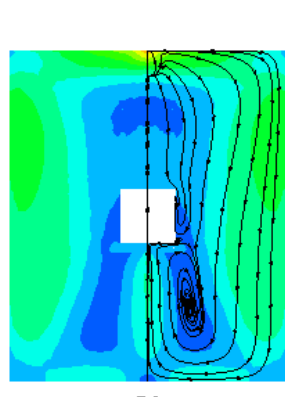

B5
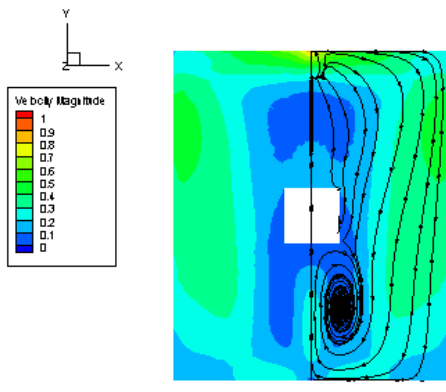

B3

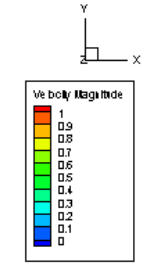

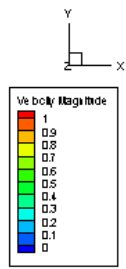

Fig.8. Velocity magnitude field of the $z=0.5 \mathrm{~m}$ plane with contaminant gas released at $\mathrm{B}(0,2 \mathrm{~m}, 0.5 \mathrm{~m})$.

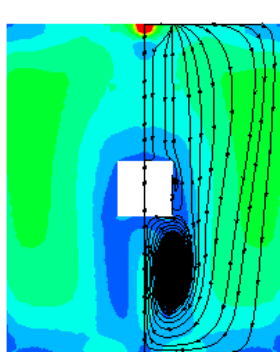

B1

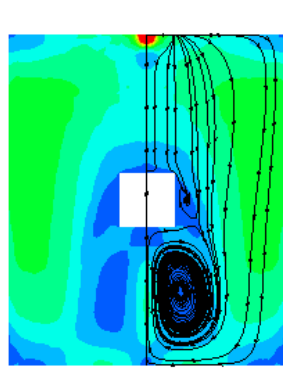

B4
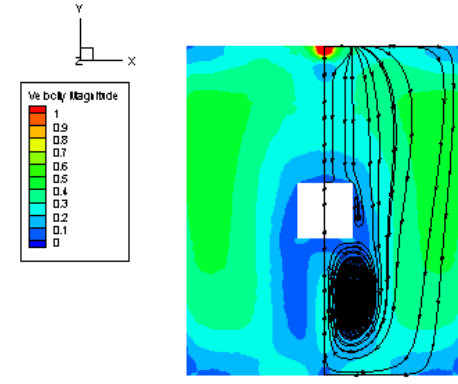

B2

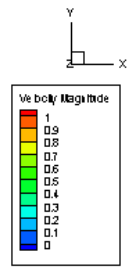

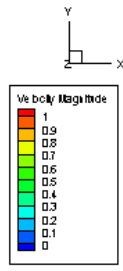

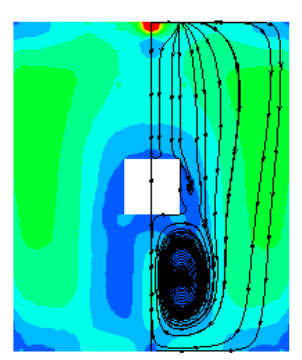

B3

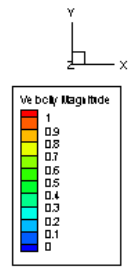

Fig.9. Velocity magnitude field of the $\mathrm{z}=0.2 \mathrm{~m}$ field with contaminant gas released at $\mathrm{B}(0,2 \mathrm{~m}, 0.5 \mathrm{~m})$.

Fig.10 gives the velocity magnitude contour of the symmetry plane. We can observe that with the increase of $\theta$, the single vortex on the A side of the obstacle changes little. On the B side of the obstacle, a new vortex appears in the vicinity of the source. 
Comparing the configurations with different source locations, we can find that when the emission rate is high to some extent, the flowfield can be affected to create a new vortex.

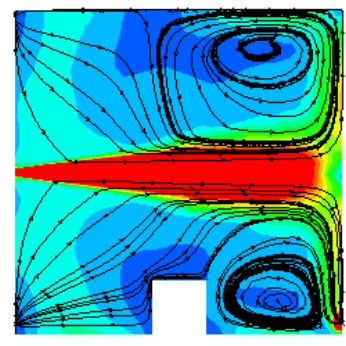

B1

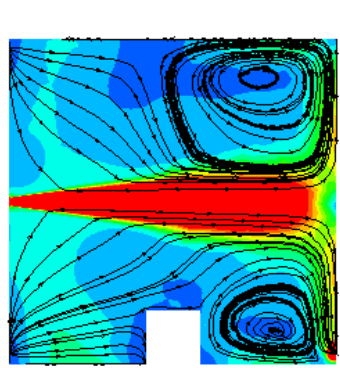

B4
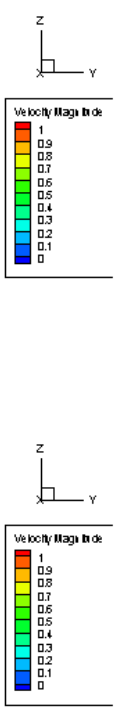

B2

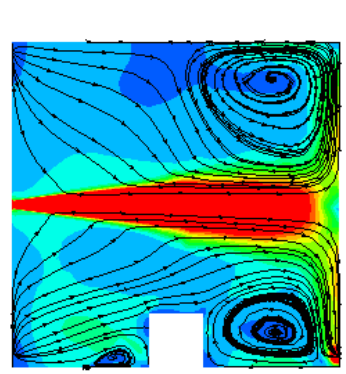

Fig.10. Velocity magnitude field of the symmetry plane with contaminant gas released at $\mathrm{B}(0,2 \mathrm{~m}$, $0.5 \mathrm{~m})$.

\subsection{Contaminant distribution}

Fig. 11 gives the iso-surface of $C^{*}=2$ (the blue one) and the iso-surface of 2000ppm (the red one) when the source is at $\mathrm{A}(0,4 \mathrm{~m}, 0.5 \mathrm{~m})$. It is obvious that with the increase of $\theta$, the isosurface of $C^{*}=2$ first enlarges, then starts to fall toward the floor like liquid. The shape of isosurface $C^{*}=2$ changes totally from A4 to A5 (from $\theta=3.936$ to $\theta=5.342$ ). The flammable region is first very small, which means negligible potential risk of explosion. It is also turning noticeable from A4 to A5 (from $\theta=3.936$ to $\theta=5.342$ ).

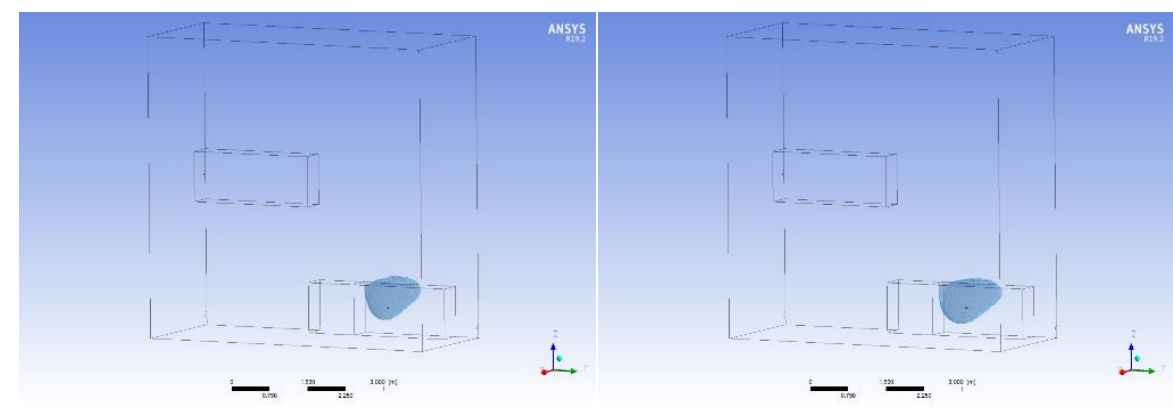



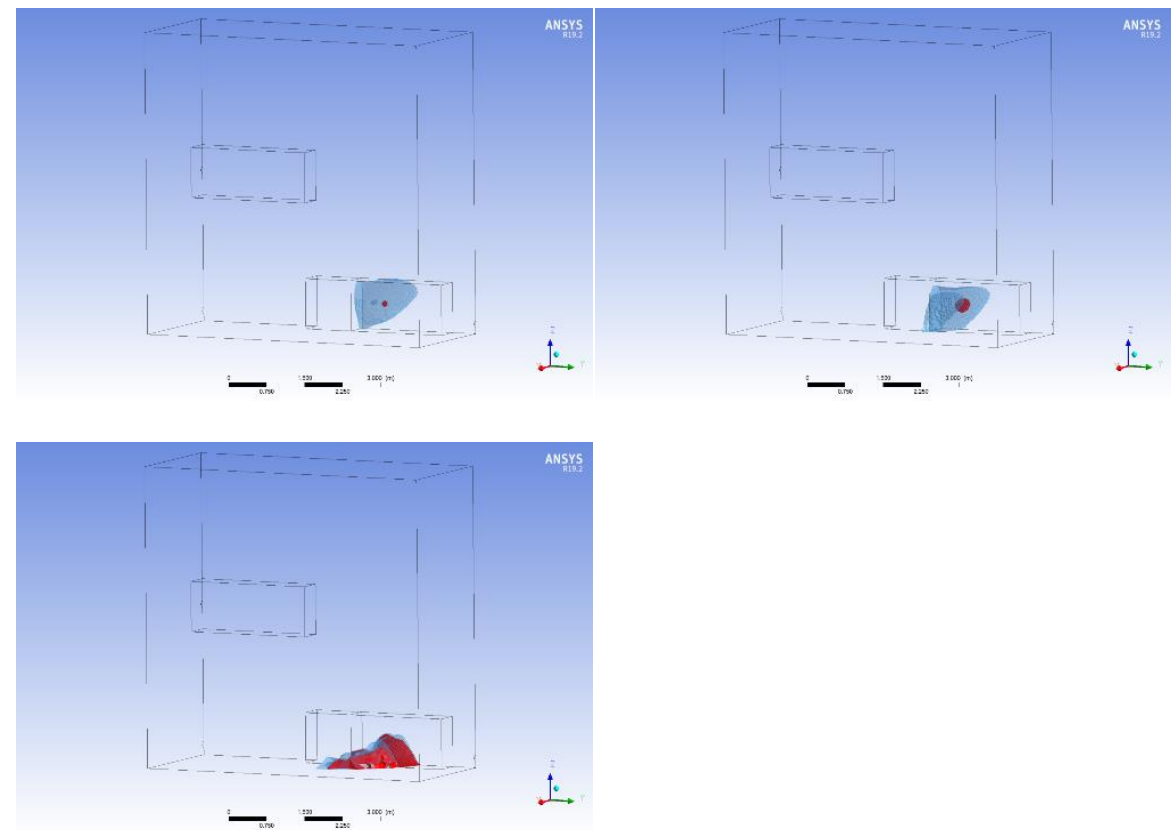

Fig.11. Iso-surface of $C^{*}=2$ and the flammable region for source at $(0,4 \mathrm{~m}, 0.5 \mathrm{~m})$.

Fig. 12 gives the iso-surface of $C^{*}=2$ (the blue one) and the iso-surface of 2000ppm (the red one) when the source is at $\mathrm{B}(0,2 \mathrm{~m}, 0.5 \mathrm{~m})$. It is obvious that with the increase of $\theta$, the iso-surface of $C^{*}=2$ first becomes larger, then starts to fall toward the floor like liquid. The iso-surface of $C^{*}=2$ changes totally from B4 to B5 (from $\theta=7.331$ to $\theta=9.950$ ). In $\mathrm{B} 5$ condition, the shape of iso-surface for $C^{*}=2$ is similar to the vortex on the $\mathrm{z}=0.2 \mathrm{~m}$ plane. The flammable region is turning noticeable from B4 to $\mathrm{B} 5$ (( from $\theta=7.331$ to $\theta=9.950$ ).

Comparing Fig.11 and Fig.12, the evolving of the iso-surface of $C^{*}=2$ and the flammable region are similar. When the emission rate increases to a certain extent, the shape of iso-surface of $C^{*}=2$ changes while the flammable region becomes noticeable. For different positions of the source, the critical dimensionless number $\theta$ lies in different ranges.

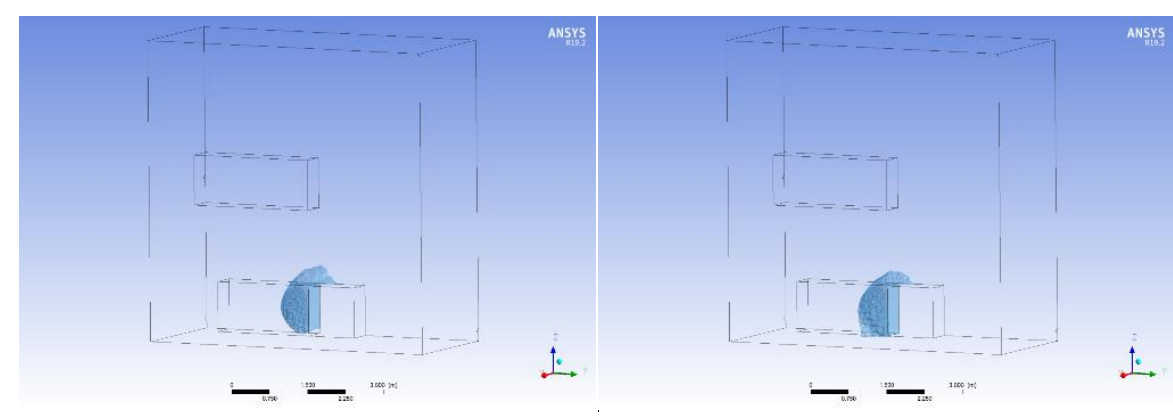



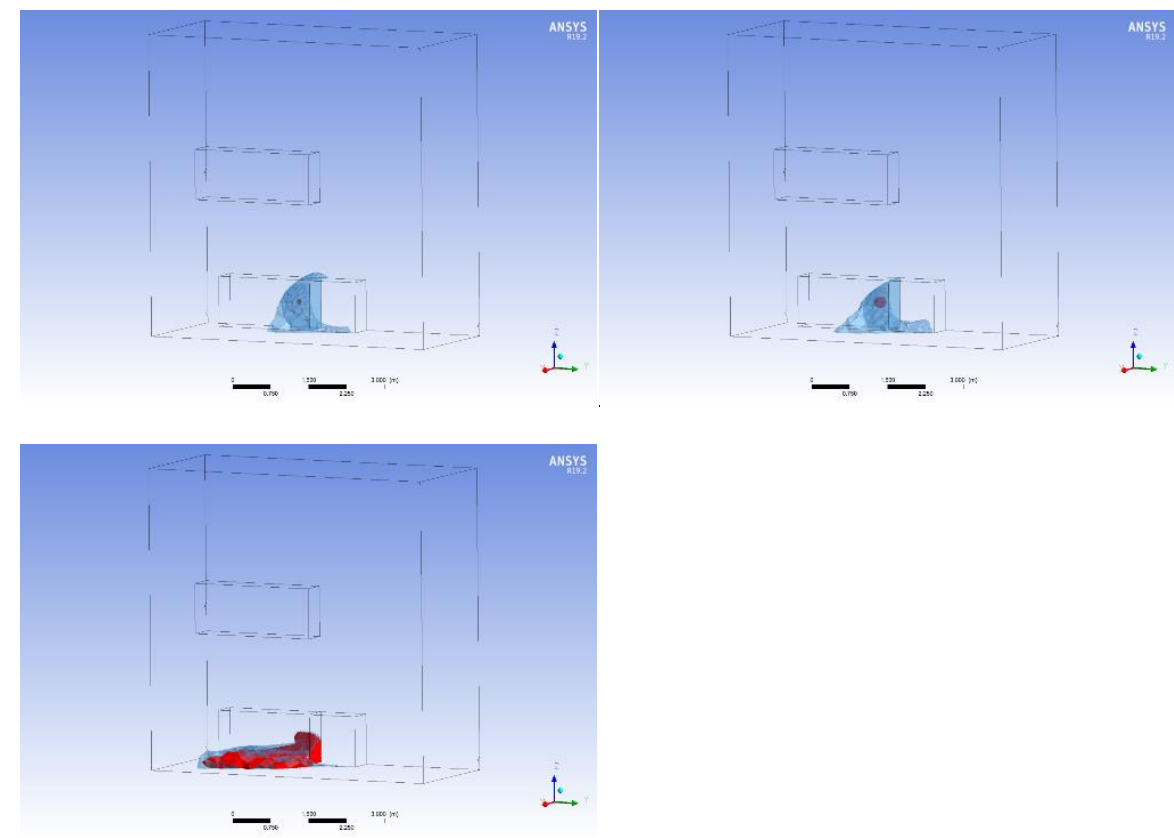

Fig.12. Iso-surface of $C^{*}=2$ and the flammable region for the source at $(0,2 \mathrm{~m}, 0.5 \mathrm{~m})$.

\section{Conclusions}

By investigating the flowfields and concentration fields with two contaminant emission positions and five emission rates, we conclude the effect of the emission rate on the flow pattern and contaminant transport pattern within a large space middle supply and low exhaust.

With the increase of the emission strength, the negative buoyancy grows. New vortexes appear when the emission rate is high enough, both when the contaminant source is on the side close to the outlet and the side far from the outlet.

With the increase of $\theta$, the center of gravity for the zone of $C^{*}>2$ becomes lower, the flammable region enlarges. With the increase of $\theta$, the flammable region becomes noticeable, which means that the space is exposed to potential risk.

\section{Acknowledgment}

This research has been supported by the China National Key R\&D Program during the 13th Five-year Plan Period (Grant No.

2018YFC0705300). This research has been supported by the National Natural Science

Foundation of China under Grant No. 51278370.

\section{References}

1 L. Ricciardi, C. Prevost, L. Bouilloux, R. Sestier-Carlin, Experimental and numerical study of heavy gas dispersion in a ventilated room, J Hazard Mater 152(2) (2008) 493-505.

2 T.G. Foat, J. Nally, S.T. Parker, Investigating a selection of mixing times for transient pollutants in mechanically ventilated, isothermal rooms using automated computational fluid dynamics analysis, Building and Environment 118 (2017) 313322.

3 L. Cheng, B.Z. Li, Q.X. Cheng, A.N. Baldwin, Y.Z. Shang, Investigations of indoor air quality of large department store buildings in China based on field measurements, Building and Environment 118 (2017) 128-143.

4 P. Rohdin, B. Moshfegh, Numerical predictions of indoor climate in large industrial premises. A comparison between different $\mathrm{k}-\varepsilon$ models supported by field 
measurements, Building and Environment 42(11) (2007) 3872-3882.

5 E. Finlayson, A. Gadgil, T. Thatcher, R. Sextro, Pollutant dispersion in a large indoor space. Part 2: Computational fluid dynamics predictions and comparison with a scale model experiment for isothermal flow, Indoor Air 14(4) (2004) 272-283.

6 Y. Tominaga, T. Stathopoulos, CFD simulations of near-field pollutant dispersion with different plume buoyancies, Building and Environment (2018).

7 Y. Tominaga, S. Akabayashi, T. Kitahara, Y. Arinami, Air flow around isolated gable-roof buildings with different roof pitches: Wind tunnel experiments and CFD simulations, Building and Environment 84 (2015) 204213.

8 S. Yin, Y.G. Li, Y.F. Fan, M. Sandberg, Unsteady large-scale flow patterns and dynamic vortex movement in near-field triple buoyant plumes, Building and Environment 142 (2018) 288-300.

9 B. Blocken, LES over RANS in building simulation for outdoor and indoor applications: A foregone conclusion?, Building Simulation 11(5) (2018) 821-870.

10 R.E. Britter, J. McQuaid, Workbook on the dispersion of dense gases, Health and Safety Executive, 1988.

11 P.V. Nielsen, F. Allard, H.B. Awbi, L. Davidson, A. Schälin, Computational Fluid Dynamics in Ventilation Design REHVA Guidebook No 10, Taylor \& Francis, 2007.

12 H.K. Versteeg, W. Malalasekera, An introduction to computational fluid dynamics: the finite volume method, Pearson Education2007.

13 R.E. Britter, Atmospheric dispersion of dense gases, Annual review of fluid mechanics 21(1) (1989) 317-344.
14 R. Britter, A review of some mixing experiments relevant to dense gas dispersion, Clarendon Press, Oxford, UK1988. 